\title{
Development of a Vacuum Ultraviolet Detector for Dark Photon Searches
}

\author{
Abaz Kryemadhi, ${ }^{a, *}$ Niklas Hellgren ${ }^{a}$ and Ryan Thurber $^{a}$ \\ ${ }^{a}$ Messiah University, \\ One University Ave, Mechanicsburg, PA, USA \\ E-mail: akryemadhi@messiah.edu
}

Dark photon arises as the extra gauge boson in a U(1) Standard Model extension and it couples to ordinary photon via kinetic mixing. The parameter space spans many orders of magnitude in energy and has been explored widely by terrestrial and astrophysical measurements. In this work, we focused on development of a detector system to study a narrow energy band from 7-8 eV motivated by other studies. The photons in this energy band have large absorption due to molecular oxygen where absorption length is of order of $\mathrm{cm}$ at atmospheric pressure, and the detection system has to be setup in vacuum or use nitrogen purging to reduce their attenuation. We constructed our detector system using low dark rate photomultipliers sensitive at these energies with aluminum reflector akin to FUNK experiment to enhance collection, and setup our experiment in a vacuum chamber. Results on performance and preliminary sensitivity will be reported.

$37^{\text {th }}$ International Cosmic Ray Conference (ICRC 2021)

July 12 th - 23rd, 2021

Online - Berlin, Germany

\footnotetext{
${ }^{*}$ Presenter
} 


\section{Introduction}

Dark photon is a natural U(1) extra gauge boson in extension to Standard Model which couples kinetically to photons. The kinetic mixing parameter space has been explored extensively over wide range of energies. The optical region has been explored by dish antenna experiments for example FUNK [3]. There is a motivation to search for vacuum UV dark photons from temperature excursion and oxygen absorption bands at these energies in upper stratosphere [4,5]. The photons at energies 7-8 eV (150-180 nm) have absorption length of $\sim \mathrm{cm}$ so even if they are produced from dark photon conversion they are absorbed before they can arrive to a photo-detector. We designed a dish antenna experiment similar to FUNK and placed it in a vacuum chamber in order to be able to detect these photons and report on performance and preliminary kinetic mixing sensitivity.

\section{Experimental Setup}

Our experiment consist of a vacuum chamber an aluminum parabolic reflector and photodetector with data acquisition. Figure 1(Left) shows a picture of the vacuum chamber and figure 1 (Right) shows a picture of the reflector inside the vacuum chamber. For the photo-detecor we use ET Enterprises Electron Tubes photo-multiplier 9107QB with sensitivity from 160-630 nm. This photomuliplier has lower dark rate and excellent single photon resolution. We determined the best operating high voltage for the photomultiplier was $1050 \mathrm{~V}$ for best single photon resolution. For the dish we used a parabolic aluminum reflector from Edmund Optics with a diameter of $30 \mathrm{~cm}$, chosen because aluminum has high reflectively in vacuum UV region. The dish had to be customized to fit inside the vacuum chamber with a total effective area of $0.05 \mathrm{~m}^{2}$. The vacuum chamber was operated at a pressure of $\sim 10^{-5}$ torr, and the light exposed areas were covered with black cloth material. This particular PMT does not operate in vacuum so it was mounted on outside viewing through a $M g F_{2}$ glass window with transmission of light in vacuum UV up to $90 \%$.

A DRS4 Evaluation board was used for data acquisition of the events.
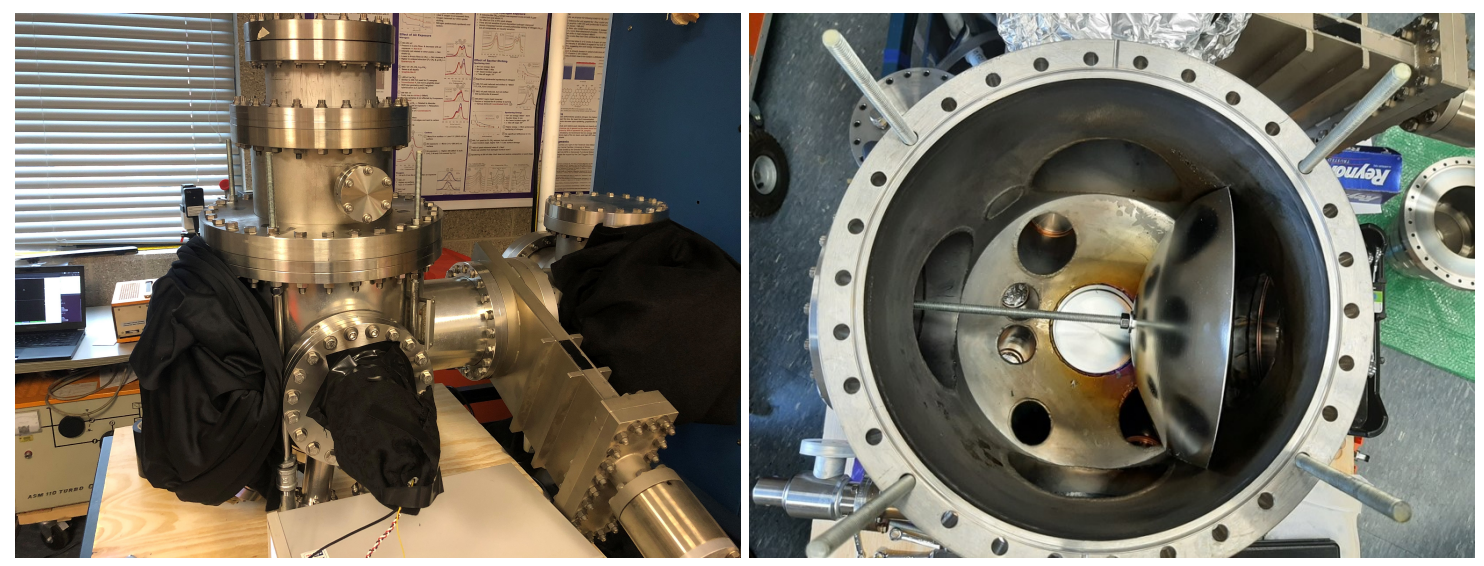

Figure 1: (Left) Vacuum Chamber with photodetector mounted on outside. (Right) A picture of parabolic mirror inside the vacuum chamber facing the PMT. 


\section{Measurements \& Analysis}

We acquired about 10 million events each for three different configurations; closed PMT for dark counts, aligned PMT with the reflector for Data, and offset PMT for a control sample. Additionally we applied quality cuts on the data to exclude events which had large RF noise, events at the beginning of run due to hysteresis. We also observed some light leak from ionization event in the cold cathode pressure gauge, although there was no direct line of sight to the PMT. We therefore excluded events when the pressure gauge was on. Figure 2 shows the trigger rate for a subset of data for the dark run and for the data run. The larger peaks appear more frequently in the data sample. For the dark run these peaks are due to Cherenkov events in the glass of the PMT. The data run has additional Cherenkov events caused in the glass of two vacuum windows.

Figure 3 shows the arrival time difference between subsequent events. The number of events for the dark and control run is normalized to the number of data events. The arrival time distribution is approximately exponential for the dark run indicating not correlated events. However for both control and data we see the appearance of a cluster at arrival times of 1-1.5 seconds. This cluster of events arriving once every 1-1.5 seconds are consistent with Cherenkov events caused by cosmic ray muons inside the glass windows of the vacuum chamber.
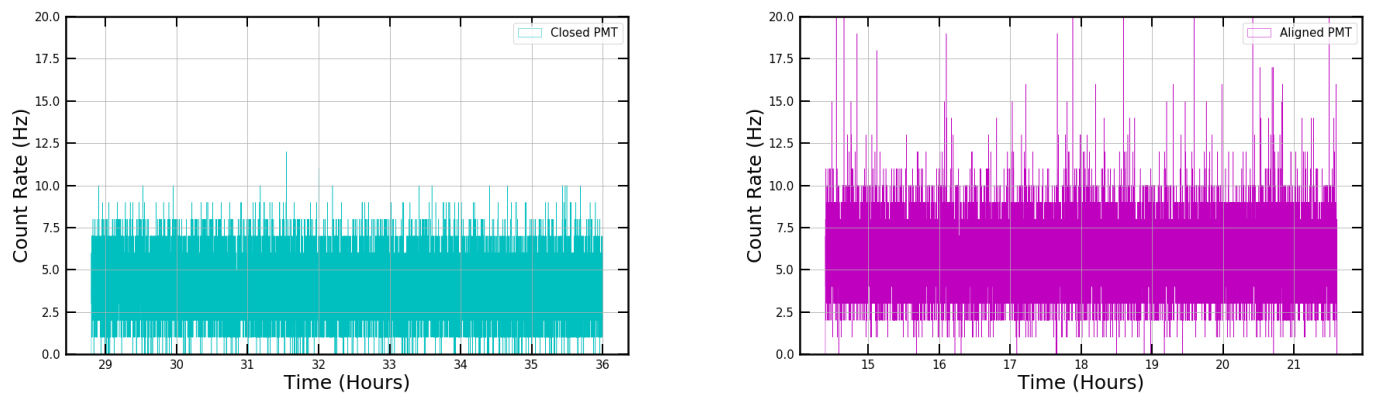

Figure 2: (Left) Trigger Rate for a subset of Dark Run. (Right) Trigger Rate for a subset of Data Run.

Figure 4 shows the charge distribution for these three different runs normalized to data event number.

Figure 5 shows the count rate difference between data and control which would be a measure of the signal. There is some modulation which is explained by that fact that in offset configuration the PMT was more exposed to Cherenkov events from the larger vacuum window. For this case a count rate of $v \sim 0.01 \mathrm{~Hz}$ determines the limit we can place on dark photon kinetic mixing. The kinetic mixing for this count rate according to $[2,3]$ is:

$$
\chi_{\text {sens }} \simeq 5.5 \times 10^{-13}\left[\left(\frac{0.1}{\eta}\right)\left(\frac{v}{\mathrm{~Hz}}\right)^{1 / 2}\left(\frac{100 \mathrm{days}}{\mathrm{T}}\right)^{1 / 2}\left(\frac{0.3}{\rho_{C D M}}\right)\left(\frac{1 m^{2}}{A_{\text {eff }}}\right)\right]^{1 / 2},
$$

where $\eta \sim 0.08$ is the efficiency, $v \sim 0.01 \mathrm{~Hz}, T$ is live-time of experiment in days ( 0.3 days) for this particular run, and $A_{\text {eff }} \sim 0.05 \mathrm{~m}^{2}$. The sensitivity is $\chi_{\text {sens }} \simeq 10^{-11}$. This preliminary sensitivity is for energy ranges in optical region in addition to vacuum UV. We plan to design a much larger area detector in larger vacuum and be able to isolate the Cherenkov events and other events and eventually carry out a more stringent search in vacuum UV energy ranges. 


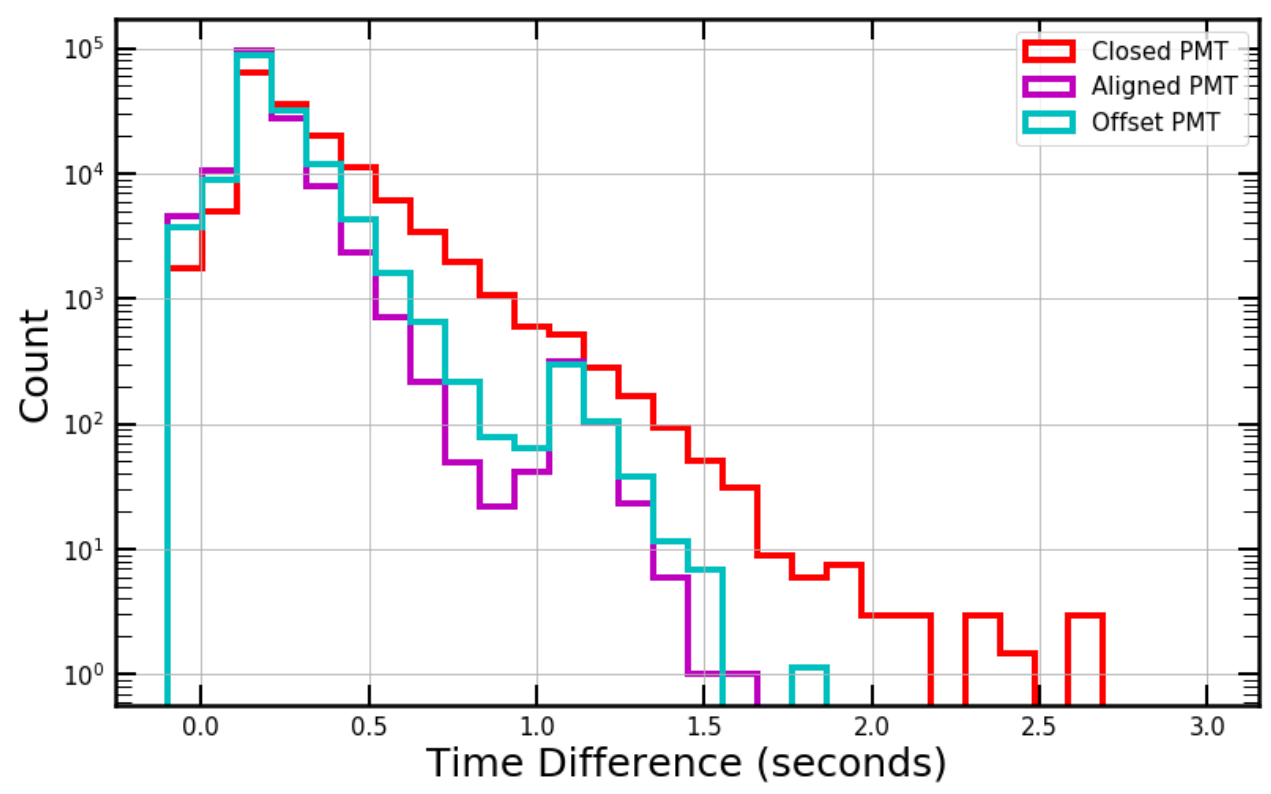

Figure 3: Arrival Time between successive events for dark, control, and data run

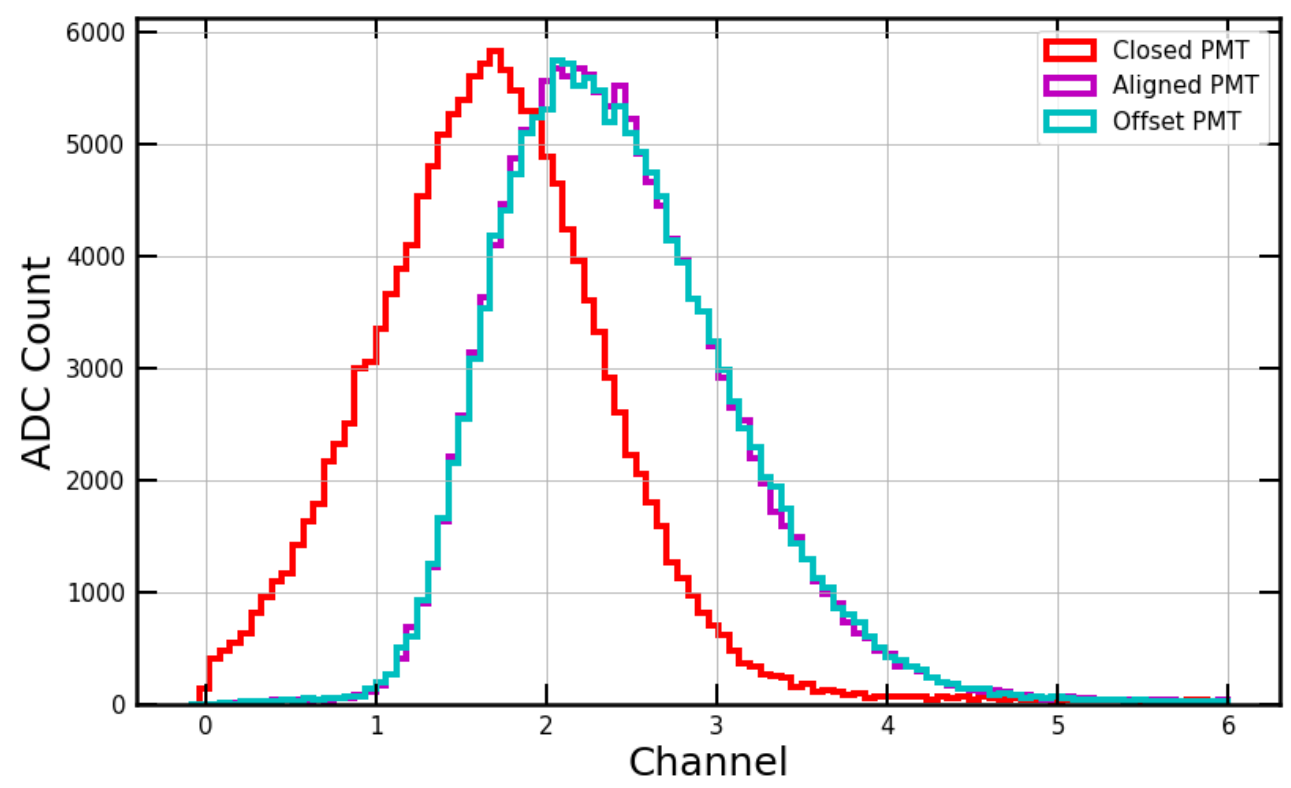

Figure 4: Charge distribution for dark, control, and data run 


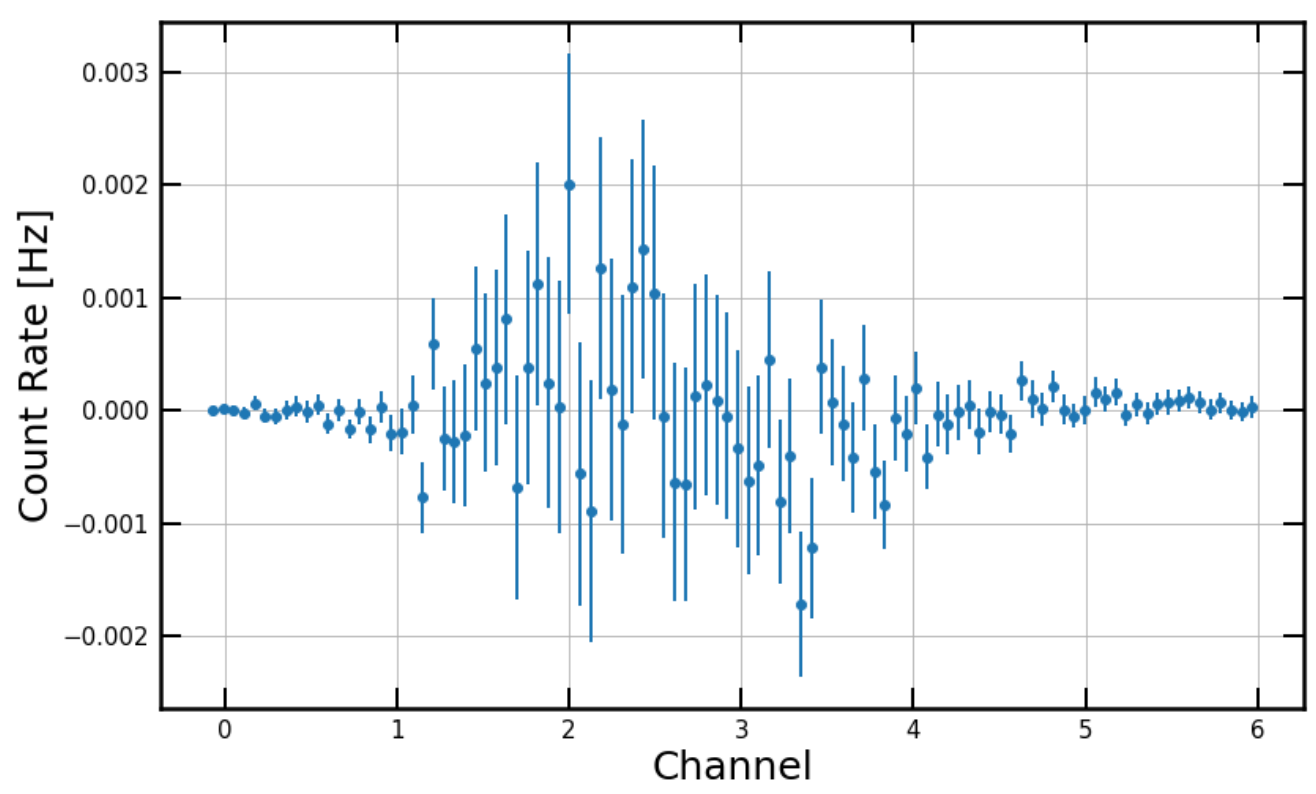

Figure 5: Difference in count between data and control

\section{Conclusion}

We constructed a dish antenna detector in vacuum to search for dark photon to photons conversions happening at the surface of a parabolic aluminum reflector which then would be detected by the PMT. The vacuum UV search was motivated by other studies for Dark Matter allowing us to study the 7-8 eV range window. In this study we included also the optical region. We learned the source of the correlated noise caused by Cherenkov events in glass and we placed a preliminary kinetic mixing limit. In future studies, we plan to collect more data, isolate the noise better, include a larger reflector and vacuum, and carry out a more detailed analysis.

\section{Acknowledgement}

We acknowledge Messiah University Workload Reallocation Program.

\section{References}

[1] J. Jaeckel, J. Redondo, An antenna for directional detection of WISPy dark matter, 2013JCAP11016, arXiv:1307.7181 [hep-ph]

[2] J. Suzuki et. al., Experimental search for hidden photon CDM in the eV mass range with a dish antenna, JCAP09(2015)042 
[3] A. Andrianavalomahefa,et al.,, Limits from the FUNK experiment on the mixing strength of hidden-photondark matter in the visible and near-ultraviolet wavelength range, PRD 102,(2020) 042001

[4] G. Cantatore et. al., On the Direct Detection of Dark Matter in the Stratosphere, ePrint:2012.03353 [hep-ex]

[5] K. Zioutas et. al, Stratospheric temperature anomalies as imprints from the dark Universe, Phys.Dark Univ. 28 (2020) 100497 